\title{
Influence of cat owners' feeding practice and attitude towards obesity on body condition of cats
}

A Kikuchi, E Bleach, S Chikunya

Writtle College, Chelmsford, Essex, United Kingdom

Email:lovelabnero@yahoo.co.jp

Introduction Several authors have reported obesity as one of the most common malnutrition disorders in cats (Anderson 1973, Crane 1991, Markwell et al., 1994, Russell et al., 2000). Successful prevention and treatment of the problem requires understanding and participation of cat owners. The majority of previous studies have investigated risk factors for obesity, but paid little attention to the feeding practices and attitudes of cat owners towards obesity. In addition to understanding the consequences of obesity, and the dietary and physiological risk factors, it is equally important to understand the opinions, attitudes and behaviour of cat owners. Therefore, the aim of this study was to investigate feeding practices, cat owners' attitude towards obesity in cats and their influence on the body condition of cats.

Materials and methods In this study, 139 cat owners, who were clients at a veterinary practice in Chelmsford (Essex, UK), with a total 201 cats, were interviewed by a questionnaire. The questionnaire comprised of three sections. The first section gathered data about the gender of cats, age, breed, body weight (actual or estimated), health status, places cats spent most of their time, whether or not they hunted, whether or not cats were fed elsewhere, level of physical activity of the cats, their living environment and existence of other animals in the household. Section two had questions pertaining to feed and feeding regimen. For instance, types and brands of cat food provided, reasons for the choice of food, frequency of feeding, methods of dish-out, amount of foods offered, adherence of owners to feeding instructions on the labels, frequency of feeding treats and milk, types and amount of milk offered, and whether or not the amount of food is adjusted. The third section gathered information about the cat owners. The information gathered included; frequency of playing with cats, experience of cat ownership, frequency of visiting veterinary practices, and amount of money spent on the cat food per week, owner's perception of body condition of their cat, owner's attitude to and knowledge of obesity. In addition, data on the owner's age, gender, height, weight, and the number of people in the household was also gathered. The body condition of the cats were categorised on a five-point scale $(5=$ obese and $1=$ very thin) (Thatcher et al., 2000) by the cat owners and the veterinarian, independently. The data collected were analysed using the chi-square test to determine whether any associations existed between each factor and the body condition scores of cats. Spearman's rank correlation test was applied to assess the strength of relationship between variables, e.g. between the amount of money spent on cat food and the body condition score of the cat etc.

Results Of the 198 cats that were condition scored by their owners; 20\%, 14\%, 37\%, 23\% and 5\% were classified as thin, underweight, optimal, overweight and obese, respectively. In comparison, body condition scores assessed by veterinarians revealed that cat owners underestimated condition scores in $34 \%$ of the cat owners surveyed. All cats in this study were fed commercial cat food by their owners. The majority were fed both dry and moist cat foods, either twice a day or on demand. The majority of cat owners did not follow cat food manufacturers' feeding instructions on the label and believed that cats can regulate their food intake. The factors which were found to have significant association with body condition score were the cats' age $(p<0.05)$, whether or not the cats hunted $(p<0.05)$, whether or not the cats were fed elsewhere $(p<0.01)$, the level of physical of activity of the cats $(p<0.05)$ and the age of cat owners $(p<0.01)$. Most cat owners had a negative attitude towards their animals' obesity, stating that obesity had a negative impact on health of cats. However, some owners (10 of 140 respondents) thought that problem of excessive weight would not happen to their cats. Owners of overweight cats were more likely to feel that it was not easy to manage weight loss treatment in their cats than owners of under and optimal weight cats $(p<0.01)$. Cats with owners younger than 60 years were more likely to be of optimal weight than those owned by the over 60 age-group $(\mathrm{p}<0.01)$. In general, cat owners younger than 30 years of age tended to adhere to manufacturers feeding instructions more than owners in older age groups $(p<0.01)$. No statistically significant association was observed between the age of cat owner and the amount of money spent on cat food. Male (32 out 38) owners were more likely to give their cats treats than female $(82$ out of 157$)$ owners $(\mathrm{p}<0.01)$.

Conclusion This study confirmed that there is a lack of knowledge and awareness of obesity in cats amongst their owners. To prevent and reduce the incidence of obesity in cats more information should be provided effectively to cat owners and individual weight management protocols should be organised by professionals.

\section{References}

Anderson, R.S. 1973. Veterinary Annual 14, 182-186.

Crane, S. W. 1991. Journal of Small Animal Practice 32, 275-282.

Markwell, P. J., Butterwick, R. F., Wills, J. M. and Raiha, M. 1994. International Journal of Obesity 18(1, Supplement), 39S-43S.

Russell, K., Sabin, R., Bradley, R. and Harper, E. J. 2000. Journal of Small Animal Practice 41, 12-17.

Thatcher, C. D., Hand, M. S. and Remillard, R. L. 2000. Small Animal Clinical Nutrition. 4th edition, 1-19. 